\title{
Parametric vibrations in mechanical systems on the example of a mining hoist installation
}

\author{
Stanistaw Wolny ${ }^{*}$ \\ AGH University of Science and Technology, Kraków, Poland
}

\begin{abstract}
This study summarises the research efforts undertaken to establish how variable stiffness of shaft steelwork should affect the amplitudes of horizontal displacements of selected components of a conveyance (skip head and skip bottom frame). Most studies investigating the dynamic behaviour of conveyances travelling in the shaft rely on a simplifying assumption whereby the shaft steelwork stiffness is taken to be constant, despite its cyclic variations. This simplification vastly facilitates further dynamic analyses, yet it is necessary to establish which parameters of the shaft steelwork behaviour should be affected, in the context of stiffness in the guiding systems. With regard to parameters of typical hoist installations currently operated in Polish collieries, the study demonstrates that the approach whereby the variable shaft steelwork and its impacts on amplitudes of horizontal displacements of selected components of a conveyance (skip head or bottom frame) should be omitted in the analysis of the hoist dynamics is fully justified and merited in practical applications.
\end{abstract}

\section{Introduction}

The dynamic behaviour of a conveyance was analysed under the normal operating conditions (conveyance ride at the fixed velocity $V=$ const) basing on the assumption that horizontal displacements of the lumped masses in the hoist model are attributable to random misalignments and irregularities of the guide strings. Finally, the spectral densities of displacements of the selected mass components were obtained [1].

The formulas derived accordingly and their graphical interpretation [2,3] should enable the selection of the system's parameters such that displacements of the selected points in the model should not exceed the critical levels specified in relevant mining regulations [4].

The dynamic analysis will yield the variance of interaction forces between a conveyance and shaft steelwork, which is requisite for effective control of the conveyance's profile in the context of its endurance parameters. In order that the results could be reliably used in practical applications, the Author undertook to validate the simplifying assumptions discussed in $[1,2,3]$. The underlying assumption in the dynamic analysis summarized in $[1,2,3]$ is the linear dependence of the damping factor $(2 \mathrm{~h})$ and stiffness $(\mathrm{k})$ of the guiding system (roller guides-shaft steelwork), see Fig 1. The guiding system comprises roller

* Corresponding author: stwolny@agh.edu.pl 
guides which, during the conveyance ride, slide on the series- connected guiding rails (beams arranged vertically and supported on buntons (horizontal beams fixed to the shaft steelwork). When the horizontal displacements of a conveyance are insignificant, the elasticity and damping factors are taken to be linear. However, when the guide rails-bunton connections are sufficiently rigid, the guide string can be treated as a beam on elastic supports, its span length $l_{\mathrm{o}}$ equal to the distance between buntons. The tenable assumption is [5] that elasticity of the shaft steelwork (buntons plus the guide rails) should be governed by the formula:

where:

$$
k_{\mathrm{z}}=\frac{k_{\mathrm{d}}+k_{\mathrm{p}}}{2}-\frac{k_{\mathrm{d}}-k_{\mathrm{p}}}{2} \cos \omega t
$$

$k_{\mathrm{z}}$ - equivalent elasticity factor of the shaft steelwork

$k_{\mathrm{d}^{-}}$equivalent elasticity factor of shaft steelwork at the point the guide rail is fixed to the bunton;

$k_{\mathrm{p}}$-equivalent elasticity factor of the shaft steelwork at mid-span between two buntons;

$\omega$ - frequency

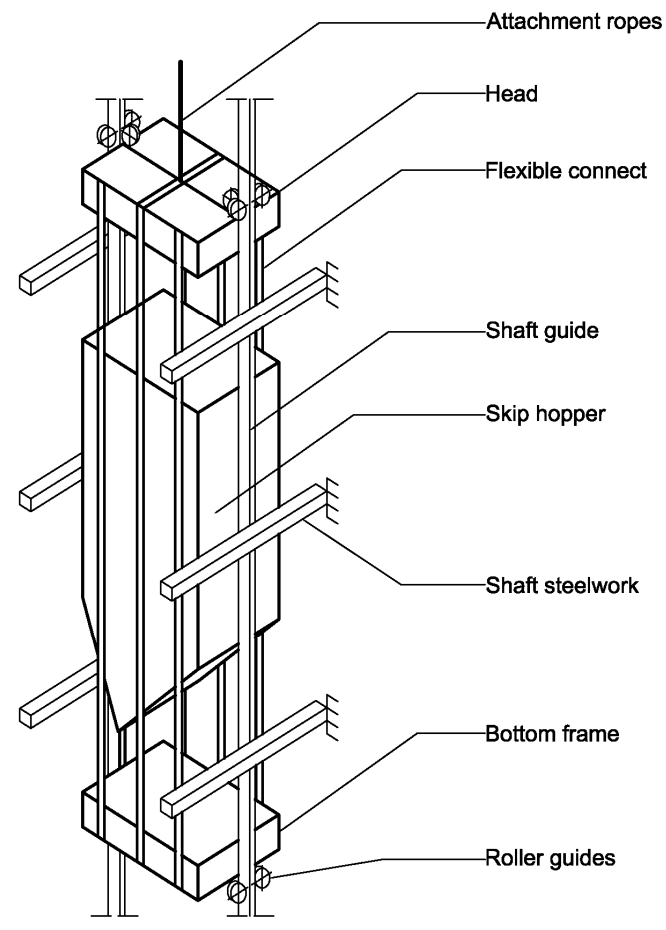

Fig 1. Schematic diagram of the hoist installation.

The assumption whereby the elasticity factor of the shaft steelwork is taken to be constant irrespective of periodic variations (1) simplifies further analysis yet it is necessary to establish to which parameters of the shaft steelwork behaviour it should be applicable in the context of rigidity in the guiding systems.

The impacts of varying shaft steelwork elasticity/stiffness on stability of vibrations in the conveyance-shaft steelwork system were investigated in the work [6]. This study summarises the Author's efforts to determine how variable stiffness of the shaft steelwork 
should affect the amplitude of horizontal displacements of selected components of the conveyance (for example roller guides).

\section{Analysis of dynamic behaviour of the conveyance [1, 2, 3]}

In order to establish how variable stiffness of the shaft steelwork should affect the amplitudes of horizontal displacements of conveyance components during its ride in the shaft, the Author recalls the results of dynamic analyses summarised in the works $[1,2,3]$.

The conveyance is represented by a triple mass model (Fig. 2) whose dynamic motion equations are based on an assumption that horizontal displacements of the modelled lumped masses are induced by random irregularities and misalignments of guide strings (indicated as $x_{1}(\mathrm{t})$ and $x_{1}(t+\tau)$ in Fig. 2).

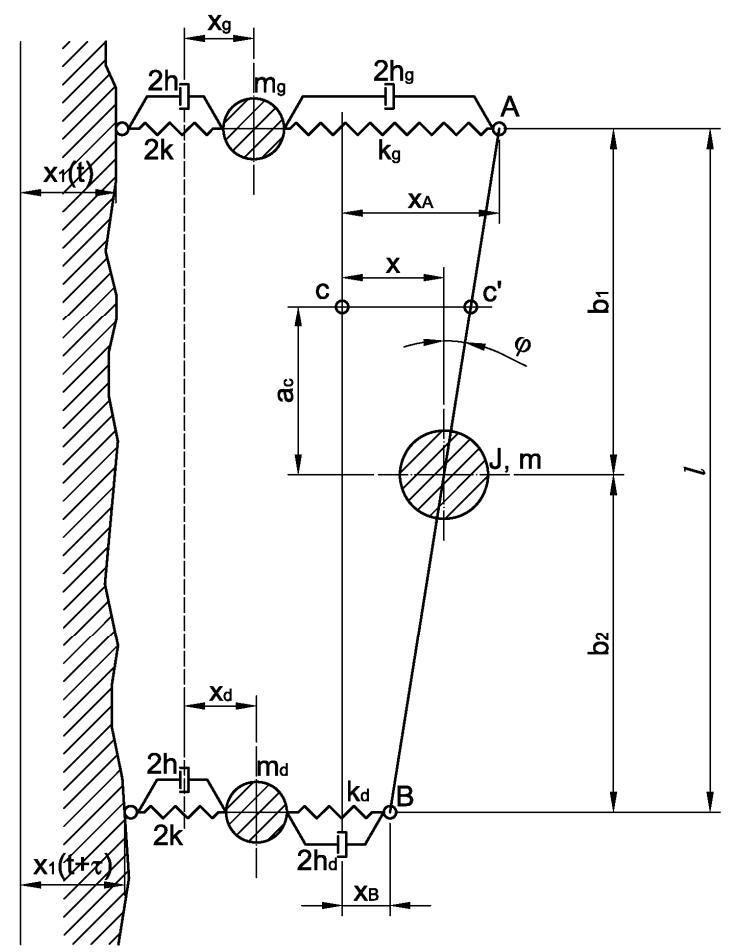

Fig 2. Mechanical model of a hoist installation.

Underlying the equations of motion of the system are the Lagrange equations of the second type. Accordingly, we get $[1,2,3]$ :

where:

$$
\begin{aligned}
& \frac{\mathrm{mb}_{2}^{2}+\mathrm{J}}{\mathrm{I}^{2}} \ddot{x}_{\mathrm{A}}+\frac{\mathrm{mb}_{1} \mathrm{~b}_{2}-\mathrm{J}}{\mathrm{I}^{2}} \ddot{x}+\mathrm{k}_{\mathrm{g}}\left(x_{\mathrm{A}}-x_{\mathrm{g}}\right)+2 \mathrm{~h}_{\mathrm{g}}\left(\dot{x}_{\mathrm{A}}-\dot{x}_{\mathrm{g}}\right)=0 \\
& \frac{\mathrm{mb}_{1}^{2}+\mathrm{J}}{\mathrm{I}^{2}} \ddot{x}_{\mathrm{B}}+\frac{\mathrm{mb}_{1} \mathrm{~b}_{2}-\mathrm{J}}{\mathrm{I}^{2}} \ddot{x}+\mathrm{k}_{\mathrm{d}}\left(x_{\mathrm{B}}-x_{\mathrm{d}}\right)+2 \mathrm{~h}_{\mathrm{d}}\left(\dot{x}_{\mathrm{B}}-\dot{x}_{\mathrm{d}}\right)=0 \\
& \mathrm{~m}_{\mathrm{g}} \ddot{x}_{\mathrm{g}}+2 \mathrm{k}\left(x_{\mathrm{g}}-x_{1}(t)\right)-\mathrm{k}_{\mathrm{g}}\left(x_{\mathrm{A}}-x_{\mathrm{g}}\right)-2 \mathrm{~h}_{\mathrm{g}}\left(\dot{x}_{\mathrm{A}}-\dot{x}_{\mathrm{g}}\right)+2 \mathrm{~h}\left(\dot{x}_{\mathrm{g}}-\dot{x}_{1}(t)\right)=0 \\
& \mathrm{~m}_{\mathrm{d}} \ddot{x}_{\mathrm{d}}-\mathrm{k}_{\mathrm{d}}\left(x_{\mathrm{B}}-x_{\mathrm{d}}\right)+2 \mathrm{k}\left(x_{\mathrm{d}}-x_{1}(t+\tau)\right)-2 \mathrm{~h}_{\mathrm{d}}\left(\dot{x}_{\mathrm{B}}-\dot{x}_{\mathrm{d}}\right)+2 \mathrm{~h}\left(\dot{x}_{\mathrm{d}}-\dot{x}_{1}(t+\tau)\right)=0
\end{aligned}
$$


$x_{\mathrm{A}}=x+\mathrm{b}_{1} \varphi, x_{\mathrm{B}}=x+\mathrm{b}_{2} \varphi$,

$\varphi$ - angle of skip hopper rotation around its centre of gravity (c.o.g)

$x$ - horizontal displacement of a hopper mass

$\mathrm{m}$ - mass of a skip hopper plus the payload

J- inertia moment of a skip hopper with payload

$\mathrm{m}_{\mathrm{g}}$ - mass of the skip head

$\mathrm{m}_{\mathrm{d}}$ - mass of the bottom frame

2h, k- (linear) damping and elasticity factors of in the guiding system (roller guides- shaft steelwork)

$2 \mathrm{~h}_{\mathrm{g}}, \mathrm{k}_{\mathrm{g}}$ - damping and elasticity factors of (lateral) flexible connector between the head and hopper

$2 h_{d}, k_{d^{-}}$damping and elasticity factors of (lateral) flexible connectors between the bottom frame and skip hopper

$x_{\mathrm{g}}, x_{\mathrm{d}}$,- horizontal displacements of the face of the skip head and bottom frame, respectively $x_{1}(t), x_{1}(t+\tau)$ - function defining the imperfections of cage guides' front surfaces

$\tau$ - time of conveyance ride along the path equal to the distance between the front shoes (on the head and the bottom frame )

$\mathrm{b}_{1}, \mathrm{~b}_{2}$ - distance between the hopper c.o.g and front shoes on the top and at the bottom, respectively

$l$ - distance between top and bottom shoes

Recalling the work of Kawulok [2], it is assumed that irregularities of the guide string $\mathrm{x}_{1}(\mathrm{t})$ and $\mathrm{x}_{1}(\mathrm{t}+\tau)$ can be treated as a random stochastic process, its spectral density expressed as:

where: $D_{\mathrm{x}_{1}}, \alpha$-respective parameters.

$$
S_{\mathrm{x}}=\frac{2 D_{\mathrm{x}_{1}} \alpha}{\alpha^{2}+\omega^{2}}\left[\mathrm{~m}^{2} \mathrm{~s}\right]
$$

Basing on the results of dynamic analysis, the variance of amplitude of the conveyance-shaft steelwork interaction forces can be derived accordingly $[5,6,7]$. In order that the results could be utilised in practical applications, the Author resolved to validate the simplifying assumptions discussed in [5, 6, 7]. In this study the Author focused on establishing the impact of variable shaft steelwork stiffness on amplitudes of horizontal displacements of selected components of the conveyance.

\section{Induced parametric vibrations}

The system of equations (2)-(5) is recalled to evaluate the impacts of variable stiffness of the shaft steelwork (Eq. 1) on amplitude of horizontal displacements of the conveyance during its ride at fixed velocity $V_{0}=$ const. The term expressing the constant elasticity of the guiding system $k$ in the system of equations (2)-(5) is replaced by the parameter $k_{\mathrm{c}}$ (expressing the sum total of the shaft steelwork elasticity $k_{\mathrm{z}}$ and that of the roller guide $k_{\mathrm{k}}$ ).

In this study the evaluation procedure is limited to a specific case whereby the condition $J=\mathrm{mb}_{1} \mathrm{~b}_{2}$ is satisfied and hence the system of equations (2)-(5) could be uncoupled yielding two independent systems of equations (2)-(3) and (4)-(5).

Accordingly, the system of equations (2)-(3) is rewritten as:

$$
\begin{gathered}
\mathrm{m}_{\mathrm{A}} \ddot{x}_{\mathrm{A}}+\mathrm{k}_{\mathrm{g}}\left(x_{\mathrm{A}}-x_{\mathrm{g}}\right)+2 \mathrm{~h}_{\mathrm{g}}\left(\dot{x}_{\mathrm{A}}-\dot{x}_{\mathrm{g}}\right)=0 \\
\mathrm{~m}_{\mathrm{g}} \ddot{x}_{\mathrm{g}}+2 k_{\mathrm{c}}\left[x_{\mathrm{g}}-x_{1}(t)\right]-\mathrm{k}_{\mathrm{g}}\left(x_{\mathrm{A}}-x_{\mathrm{g}}\right)-2 \mathrm{~h}_{\mathrm{g}}\left(\dot{x}_{\mathrm{A}}-\dot{x}_{\mathrm{g}}\right)+2 \mathrm{~h}\left[\dot{x}_{\mathrm{g}}-\dot{x}_{1}(t)\right]=0
\end{gathered}
$$

where

$\mathrm{m}_{\mathrm{A}}=\frac{\mathrm{J}+\mathrm{mb}_{2}^{2}}{1^{2}}$, 
$k_{\mathrm{c}^{-}}$total elasticity factor of the whole system comprising the roller guides and shaft steelwork.

The total elasticity factor of the whole system comprising the roller guides and shaft steelwork (for the guiding elements connected in series) becomes:

where

$$
k_{\mathrm{c}}=\frac{E \cdot C\left(1-\frac{D}{C} \cos \omega t\right)}{(E+C)\left(1-\frac{D}{E+C} \cos \omega t\right)},
$$

$\frac{k_{\mathrm{d}}+k_{\mathrm{p}}}{2}=C ; \frac{k_{\mathrm{d}}-k_{\mathrm{p}}}{2}=D ; k_{\mathrm{k}}=E$ - elasticity factor of the roller guide.

After respective substitutions we get:

$k_{\mathrm{p}}=\frac{E \cdot C}{E+C} ; \varepsilon_{1}=\frac{D}{E \cdot C} ; \varepsilon_{2}=\frac{D}{E+C}$,

finally, (8) becomes:

$$
\mathrm{k}_{\mathrm{c}}=\mathrm{k}_{\mathrm{p}} \frac{1-\varepsilon_{1} \cos \omega \mathrm{t}}{1-\varepsilon_{2} \cos \omega \mathrm{t}}
$$

The values of $\varepsilon_{1}, \varepsilon_{2}$ are much less than one, accordingly, the formula (9) can be rewritten as:

where:

$$
k_{\mathrm{p}} \frac{1-\varepsilon_{1} \cos \omega t}{1-\varepsilon_{2} \cos \omega t} \approx k_{\mathrm{p}}(1+\varepsilon \cos \omega t)
$$

$\varepsilon=\varepsilon_{2}-\varepsilon_{1}$ or $\varepsilon=\frac{D}{C}-\frac{D}{E+C}=\frac{E D}{C(E+C)}$.

Finally, (9a) becomes:

where:

$$
k_{\mathrm{c}}=k_{\mathrm{p}}\left(1+\mathcal{E} \cos 2 \pi \frac{V_{0}}{l_{0}} t\right)
$$

$V_{0}[\mathrm{~m} / \mathrm{s}]$-steady -state velocity of the conveyance travel;

$l_{0}[\mathrm{~m}]$ - distance between bunton;

The underlying assumption is that vibrations of the modelled system governed by the equation (7) have been induced by kinematic displacement inputs

$x_{1}(t)=B \cdot f_{(t)}$,

where:

$f_{(\mathrm{t})}=f_{0}+f_{2} \cos \left(2 \pi \frac{V_{0}}{l_{0}} t\right)$

Substituting (11) into the system of equations (7) yields:

$$
\begin{gathered}
\mathrm{m}_{\mathrm{A}} \ddot{x}_{\mathrm{A}}+\mathrm{k}_{\mathrm{g}}\left(x_{\mathrm{A}}-x_{\mathrm{g}}\right)+2 \mathrm{~h}_{\mathrm{g}}\left(\dot{x}_{\mathrm{A}}-\dot{x}_{\mathrm{g}}\right)=0 \\
\mathrm{~m}_{\mathrm{g}} \ddot{x}_{\mathrm{g}}+2 \mathrm{k}_{\mathrm{p}}\left(1+\varepsilon \cos 2 \pi \frac{V_{0}}{l_{0}} t\right) x_{\mathrm{g}}-2 \mathrm{k}_{\mathrm{p}}\left(1+\varepsilon \cos 2 \pi \frac{V_{0}}{l_{0}} t\right)-2 \mathrm{~h}_{\mathrm{p}}\left(1+\varepsilon \cos 2 \pi \frac{V_{0}}{l_{0}} t\right)+x_{1}(t)+ \\
-\mathrm{k}_{\mathrm{g}}\left(x_{\mathrm{A}}-x_{\mathrm{g}}\right)-2 \mathrm{~h}_{\mathrm{g}}\left(\dot{x}_{\mathrm{A}}-\dot{x}_{\mathrm{g}}\right)+2 \mathrm{~h} \dot{x}_{\mathrm{g}}+2 \mathrm{~h} \frac{\mathrm{d} x_{1}}{\mathrm{~d} t}=0
\end{gathered}
$$

In terms of dimensionless parameters, $\tau=\mathrm{p}_{10} t$ in the system of equations (12), where $\mathrm{p}_{10}$ is the natural vibration frequency of the mass $\mathrm{m}_{\mathrm{A}}$ (for $\mathrm{m}_{\mathrm{g}}=0$ ). Substituting:

$$
\begin{gathered}
X_{\mathrm{A}}(t)=u(\tau), \\
X_{\mathrm{g}}(t)=v(\tau), \\
X_{1}(t)=\omega(\tau)=B\left[f_{0}+f_{2} \cos \left(2 \pi \frac{V_{0}}{l_{0}} \cdot \frac{\tau}{\mathrm{p}_{10}}\right)\right],
\end{gathered}
$$

rearranging and dividing the first equation in the system of equations (12) by $m_{\mathrm{A}} \mathrm{p}_{10}{ }^{2}$ and the second equation by $m_{\mathrm{g}} \mathrm{p}_{10}{ }^{2}$, we obtain: 


$$
\begin{gathered}
\ddot{u}+\frac{2 \mathrm{~h}_{\mathrm{g}}}{\mathrm{m}_{\mathrm{A}} \mathrm{p}_{10}} \dot{u}+\frac{\mathrm{k}_{\mathrm{g}}}{\mathrm{m}_{\mathrm{A}} \mathrm{p}_{10}{ }^{2}} u-\frac{2 \mathrm{~h}_{\mathrm{g}}}{\mathrm{m}_{\mathrm{A}} \mathrm{p}_{10}} \dot{v}-\frac{\mathrm{k}_{\mathrm{g}}}{\mathrm{m}_{\mathrm{A}} \mathrm{p}_{10}{ }^{2}} v=0 \\
\ddot{v}+\frac{2\left(h+h_{\mathrm{g}}\right)}{\mathrm{m}_{\mathrm{g}} \cdot \mathrm{p}_{10}} \dot{\mathrm{v}}+\left[2 \mathrm{k}_{\mathrm{p}}\left(1+\mathcal{E} \cos 2 \pi \frac{V_{0}}{l_{0}} \frac{\tau}{\mathrm{p}_{10}}\right)+\mathrm{k}_{\mathrm{g}}\right] \cdot \frac{1}{\mathrm{~m}_{\mathrm{g}} \mathrm{p}_{10}{ }^{2}} v-\frac{2 h_{\mathrm{g}}}{\mathrm{m}_{\mathrm{g}} \mathrm{p}_{10}} \dot{u}-\frac{\mathrm{k}_{\mathrm{g}}}{\mathrm{m}_{\mathrm{g}} \mathrm{p}_{10}} u+\frac{2 \mathrm{~h}_{\mathrm{g}}}{\mathrm{m}_{\mathrm{A}} \mathrm{p}_{10}} . \\
\cdot B \cdot f_{2} \cdot 2 \pi \frac{V_{0}}{l_{0}} \frac{1}{\mathrm{p}_{10}} \sin \left(2 \pi \frac{V_{0}}{l_{0}} \cdot \frac{\tau}{\mathrm{p}_{10}}\right)-2 \mathrm{k}_{\mathrm{p}}\left(1+\mathcal{E} \cos 2 \pi \frac{V_{0}}{l_{0}} t\right) B\left[f_{0}+f_{2} \cos \left(2 \pi \frac{V_{0}}{l_{0}} \cdot \frac{\tau}{\mathrm{p}_{10}}\right)\right] \cdot \frac{1}{\mathrm{~m}_{\mathrm{g}} \mathrm{p}_{10}{ }^{2}}=0
\end{gathered}
$$

After respective substitutions

We get

$$
\begin{aligned}
n_{1}=\frac{\mathrm{m}_{\mathrm{A}}}{\mathrm{m}_{\mathrm{g}}} ; n_{2}= & \frac{h}{\mathrm{~m}_{\mathrm{g}} \cdot \mathrm{p}_{10}} ; n_{3}=\frac{\mathrm{k}_{\mathrm{p}}}{\mathrm{k}_{\mathrm{g}}} ; n_{4}=2 \pi \cdot \frac{v_{0}}{\mathrm{l}_{0}} \cdot \frac{1}{\mathrm{p}_{10}} ; \mathrm{p}_{10}=\sqrt{\frac{\mathrm{k}_{\mathrm{g}}}{\mathrm{m}_{\mathrm{A}}}}, \\
& \frac{h_{\mathrm{g}}}{h} \ll 0, h_{\mathrm{g}}+h \cong h \text { and } h_{\mathrm{g}}=0
\end{aligned}
$$

$$
\begin{aligned}
& \ddot{\mathrm{u}}+\mathrm{u}-v=0 \\
& \ddot{v}+2 n_{2} v+n_{1}\left[1+2 n_{3}\left(1+\mathcal{E} \cos n_{4} \tau\right)\right] v-n_{1} u=2 n_{1} n_{3} B\left(1+\mathcal{E} \cos n_{4} \tau\right)\left(f_{0}+f_{2} \cos n_{4} \tau\right)+ \\
&-2 n_{2} n_{4} B f_{2} \sin n_{4} \tau
\end{aligned}
$$

To obtain the maximal amplitudes of displacement, damping in the system is neglected $\left(n_{2}=0\right)$ and hence the system of equations (15) becomes:

where:

$$
\begin{gathered}
\ddot{u}+u-v=0 \\
\ddot{v}+n_{1}\left[1+2 n_{3}\left(1+\mathcal{E} \cos n_{4} \tau\right)\right] v-n_{1} u=f_{01}+f_{02} \cos n_{4} \tau+f_{03} \cos 2 n_{4} \tau
\end{gathered}
$$

$$
f_{01}=n_{1} n_{3} B\left(2 f_{0}+\mathcal{E} f_{2}\right), f_{02}=2 n_{1} n_{3} B\left(f_{0}+f_{2}\right), f_{03}=n_{1} n_{3} B f_{2} \cdot \mathcal{E} \text {. }
$$

Solution to the approximate system of equations is given as [7]:

$$
\begin{gathered}
u=A_{0}+A_{1} \sin n_{4} \tau+B_{1} \cos n_{4} \tau \\
v=B_{0}+A_{2} \sin n_{4} \tau+B_{2} \cos n_{4} \tau
\end{gathered}
$$

Respective values of these parameters are:

$$
\begin{aligned}
& A_{0}=B_{0}=\frac{f_{01}}{2 n_{1} n_{3}}-\frac{\varepsilon}{2} \frac{f_{02}-\varepsilon f_{01}}{n_{1}+n_{1} n_{3}\left(2-\varepsilon^{2}\right)-n_{4}^{2}} \cdot \frac{\mathrm{m}}{\mathrm{p}} \\
& A_{1}=n_{4}^{2} \frac{n_{1}+2 n_{1} n_{3}-n_{4}^{2}}{2 n_{1} n_{3}-n_{4}^{2}} \cdot \frac{f_{02}-\varepsilon f_{01}}{n_{1}+n_{1} n_{3}\left(2-\varepsilon^{2}\right)-n_{4}^{2}} \cdot \frac{1}{\mathrm{p}} \\
& A_{2}=\frac{n_{1} n_{4}^{2}}{2 n_{1} n_{3}-n_{4}^{2}} \cdot \frac{f_{02}-\varepsilon f_{01}}{n_{1}+n_{1} n_{3}\left(2-\varepsilon^{2}\right)-n_{4}^{2}} \cdot \frac{1}{\mathrm{p}} \\
& B_{1}=\frac{f_{02}-\varepsilon f_{01}}{n_{1}+n_{1} n_{3}\left(2-\varepsilon^{2}\right)-n_{4}^{2}} \cdot \frac{1}{\mathrm{p}} \\
& B_{2}=\frac{f_{02}-\varepsilon f_{01}}{n_{1}+n_{1} n_{3}\left(2-\varepsilon^{2}\right)-n_{4}^{2}} \cdot \frac{\mathrm{m}}{\mathrm{p}}
\end{aligned}
$$

where:

$$
\mathrm{p}=\mathrm{m}-\frac{n_{1}}{n_{1}+n_{1} n_{3}\left(2-\varepsilon^{2}\right)-n_{4}^{2}}, \mathrm{~m}=1-n_{4}^{2} \frac{n_{1}+2 n_{1} n_{3}-n_{4}^{2}}{2 n_{1} n_{3}-n_{4}^{2}} .
$$

Accordingly, the solution becomes:

$$
\begin{aligned}
& \mathrm{u}=A_{0}+A_{1} \sin n_{4} \tau+B_{1} \cos n_{4} \tau= \\
& =\frac{f_{01}}{2 n_{1} n_{3}}-\frac{\varepsilon}{2} \frac{f_{02}-\varepsilon f_{01}}{n_{1}+n_{1} n_{3}\left(2-\varepsilon^{2}\right)-n_{4}^{2}} \cdot \frac{\mathrm{m}}{\mathrm{p}}+\frac{f_{02}-\varepsilon f_{01}}{n_{1}+n_{1} n_{3}\left(2-\varepsilon^{2}\right)-n_{4}^{2}} \cdot \frac{\mathrm{q}}{\mathrm{p}} \sin \left[n_{4} \tau+\operatorname{arctg} \varphi\right]
\end{aligned}
$$

and 


$$
\begin{gathered}
v=B_{0}+A_{2} \sin n_{4} \tau+B_{2} \cos n_{4} \tau= \\
=\frac{f_{01}}{2 n_{1} n_{3}}-\frac{\varepsilon}{2} \frac{f_{02}-\varepsilon f_{01}}{n_{1}+n_{1} n_{3}\left(2-\varepsilon^{2}\right)-n_{4}^{2}} \cdot \frac{\mathrm{m}}{\mathrm{p}}+\frac{f_{02}-\varepsilon f_{01}}{n_{1}+n_{1} n_{3}\left(2-\varepsilon^{2}\right)-n_{4}^{2}} \cdot \frac{\mathrm{r}}{\mathrm{p}} \sin \left[n_{4} \tau+\operatorname{arctg} \psi\right]
\end{gathered}
$$

where:

$$
\begin{aligned}
& \mathrm{q}=\sqrt{\left[n_{4}^{2} \frac{n_{1}+2 n_{1} n_{3}-n_{4}^{2}}{2 n_{1} n_{3}-n_{4}^{2}}\right]^{2}+1} ; \quad \mathrm{r}=\sqrt{\left[\frac{n_{1} n_{4}^{2}}{2 n_{1} n_{3}-n_{4}^{2}}\right]^{2}+\mathrm{m}^{2}} \\
& \varphi=\operatorname{arctg}\left[\frac{1}{n_{4}^{2}} \frac{2 n_{1} n_{3}-n_{4}^{2}}{n_{1}+2 n_{1} n_{3}-n_{4}^{2}}\right] ; \quad \psi=\operatorname{arctg}\left[\mathrm{m} \frac{2 n_{1} n_{3}-n_{4}^{2}}{n_{1} n_{4}^{2}}\right] .
\end{aligned}
$$

The impacts of variable stiffness of the shaft steelwork on amplitude of horizontal displacements of the skip head under parametric vibration excitations are illustrated in Fig 3,4 .

Fig. 3 plot the amplitudes of skip head displacement (v) as a function $\varepsilon=0.01 ; 0.1 ; 0.5$ expressing the periodically varying shaft steelwork stiffness, given the remaining dimensionless parameters $\mathrm{n}_{1}, \mathrm{n}_{3}, \mathrm{n}_{4}$ of the investigated system (damping $\mathrm{n}_{2}$ has been neglected to obtain the maximal displacement values).

The range of numerical values of parameters $n_{1}, n_{3}$ and $n_{4}$ was chosen such that they should be relevant and applicable to typical hoisting installations currently designed and operated in Poland [8].

In accordance with relevant mining regulations [4, 5], the respective values of parameters of the applied excitations considered in the analysis were taken as follows: $B=5$ $\mathrm{mm}, f_{0}=1, f_{2}=\varepsilon$,

\section{Conclusions}

As mentioned in earlier sections, displacements in the conveyance guiding system are induced by the conveyance-shaft steelwork interaction forces, which are of key importance in the context of fatigue endurance of the load-bearing components in the system.

Within the framework of endurance analysis, at the stage of dimensioning the magnitudes and configuration of all loads acting upon the designed structure should be precisely determined. That means that the physical model underlying the stress analysis ought to capture all relevant aspects, including the impacts of varying shaft steelwork stiffness on amplitudes of horizontal displacements of the skip components (skip head and bottom frame), which were omitted in the dynamic analyses summarised in the works [2, 3]. Plots in Fig. 3 and 4 indicate that the omission of variable shaft steelwork stiffness and its impacts on skip head displacements is revealed for $\varepsilon \geq 0.1$ whilst in typical hoisting installations currently operated in Poland the values of this parameter are less than 0.01 $(\varepsilon \leq 0.01)[8,9]$.

Thus, that the approach whereby the varying shaft steelwork stiffness and its impacts on amplitudes of horizontal displacements of selected skip components (skip head, bottom frame) should be omitted in the analysis of hoist dynamics appears to be fully justified and merited in practical applications. 


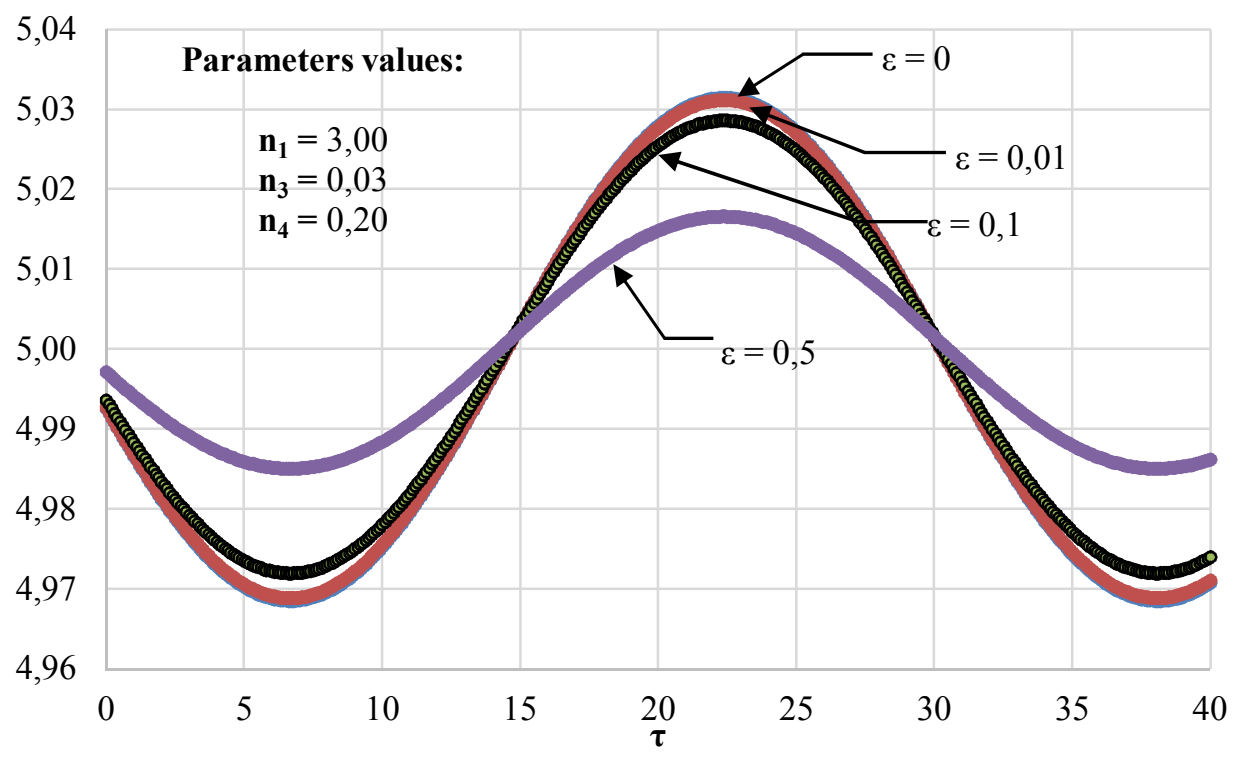

Fig 3. Selected sections of the plot of the skip head displacement $v(\tau)$ in the function $\varepsilon$, for $n 1=3.0$; $n 3=0.03 ; n 4=0.2$.

\section{References}

1. S. Wolny, Loads experienced by load-bearing components of mine hoist installations due to random irregularities and misalignments of the guide string, Journal of Machine Construction and Maintenance, vol. 110, iss. 3,p.79-86 (2018)

2. S. Wolny, Interactions in Mechanical Systems Due to Random Inputs on the example of a Mine Hoist, International Education and Research Journal, vol. 1. iss.5,p.72-76 (2015)

3. S. Wolny, Displacements in Mechanical System Due to Random Inputs in a Mine Hoist Installation, Engineering Transactions. Engng. Trans. 65,3,p.513-522 (2017)

4. Mining Regulation: The Council of Ministers regulation of 30 April 2004 in the admission of using in mining plants (Dz.U. No. 99, item 1003 of 2005 No. 80, item 695 and of 2007 No. 249, item 1853, p.1.2 skip hopper).

5. S. Kawulok, The influence of the shaft steelwork on mechanics of guiding skip hopper, GIG works, Katowice (1989)

6. S. Wolny, A. Drzewosz, Stability in mechanical system on the example of mine hoist installation (Article submitted for publication in Archives of Mining Sciences) (2018)

7. R. Gutowski, W. A. Swietlicki, Dynamics and vibrations of mechanical systems, PWN, Warsaw (1986)

8. S. Wolny et al., Developing critiria for assessing the supporting structure skip hopper of hoist installation in the terms of extending the period of safe operation, Scientific an reasearch work, Department of Strength of Materials and Structures, AGH, Cracow (2003)

9. S. Wolny et al., Development of multi-option modernization of mining cage lift $R$ $V I I$, Scientific and research work, Department of Strength and Fatigue of Materials and Structures, AGH, Cracow (2011) 